\title{
Effect of aortic taper on patterns of blood flow and wall shear stress in rabbits: association with age
}

\author{
Véronique Peiffer ${ }^{a, b}$, Ethan M. Rowland ${ }^{b, a}$, Stephanie G. \\ Cremers $^{b}$, Peter D. Weinberg ${ }^{b, c}$, Spencer J. Sherwin ${ }^{a}$ \\ ${ }^{a}$ Department of Aeronautics, Imperial College London, South Kensington \\ Campus, London, SW7 2AZ, United Kingdom \\ ${ }^{\mathrm{b}}$ Department of Bioengineering, Imperial College London, South Kensington \\ Campus, London, SW7 2AZ, United Kingdom \\ ${ }^{\mathrm{c}}$ Corresponding author (p.weinberg@imperial.ac.uk)
}

\begin{abstract}
Objective - The distribution of atherosclerotic lesions changes with age in human and rabbit aortas. We investigated if this can be explained by changes in patterns of blood flow and wall shear stress.

Methods - The luminal geometry of thoracic aortas from immature and mature rabbits was obtained by micro-CT of vascular corrosion casts. Blood flow was computed and average maps of wall shear stress were derived. Results - The branch anatomy of the aortic arch varied widely between animals. Wall shear was increased downstream and to a lesser extent upstream of intercostal branch ostia, and a stripe of high shear was located on the dorsal descending aortic wall. The stripe was associated with two vortices generated by aortic arch curvature; their persistence into the descending aorta depended on aortic taper and was more pronounced in mature geometries. These results were not sensitive to the modelling assumptions.

Conclusions - Blood flow characteristics in the rabbit aorta were affected by the degree of taper, which tends to increase with age in the aortic arch and strengthens secondary flows into the descending aorta. Previouslyobserved lesion distributions correlated better with high than low shear, and age-related changes around branch ostia were not explained by the flow patterns.
\end{abstract}

Key words: Atherosclerosis, Rabbit, Aorta, Computational Fluid Dynamics, Shear stress, Haemodynamics, Age

\section{INTRODUCTION}

Atherosclerotic lesions develop non-uniformly within the human arterial system, particularly in regions of high curvature and branching. Their distribution has been observed to change with age: in the descending thoracic aorta, lipid deposition is most prevalent downstream of intercostal branch ostia in neonates ${ }^{1}$ but lateral and upstream locations are athero-prone in adults. ${ }^{2,3}$ Although this dependence on age does not hold for all species, a similar shift has been observed in rabbits. ${ }^{4}$ Early lesions predominantly develop in a triangular region downstream of aortic branch orifices in immature rabbits, while this region is essentially spared of lesions in mature rabbits. ${ }^{5}$ Furthermore, away from branches in the descending aorta of hypercholesterolaemic rabbits, lesions occur as longitudinal or spiral stripes; this pattern is more obvious in mature than in immature vessels. ${ }^{6}$

The focal nature of atherosclerotic disease has been attributed to local variation in arterial wall properties and/or blood flow characteristics such as wall shear stress (WSS) $;{ }^{7}$ the age-related changes in lesion distribution provide a framework to test these hypotheses. A number of studies have already investigated age-related differences in aortic wall transport properties around intercostal branch openings and 
concluded that the evolution with age of short-term and quasi-steady albumin uptake patterns is similar to the evolution of the lesion distribution. ${ }^{8,9}$ Motivated by the observation that endothelial cell and nuclear elongation are shear-dependent, ${ }^{10}$ Bond et al. ${ }^{11}$ examined these parameters in the aortas of young and old rabbits. Their data suggested a positive correlation of WSS with lesion prevalence, in contrast to the current consensus that low or low oscillatory WSS promotes plaque formation. ${ }^{7,12}$

Computational Fluid Dynamics (CFD) is widely used in the field of bioengineering to compute arterial WSS. ${ }^{13-15}$ It has been used in this context to study flow in an idealised model of a single intercostal artery branching off the thoracic aorta ${ }^{16}$ and in a geometrically accurate model of one (mature) rabbit thoracic aorta, ${ }^{17}$ but it has not been applied to evaluate age-related changes in blood flow characteristics. The purpose of the study presented here was to simulate blood flow in anatomically realistic models of thoracic aortas from immature and mature rabbits, using tools described by Vincent et al., ${ }^{17}$ and to statistically compare WSS distributions in this animal model with previously-presented data on lesion prevalence. ${ }^{6}$

\section{METHODS (detailed methods are supplemented online)}

\subsection{Luminal surface reconstruction}

Aortic geometries of 5 immature and 5 mature rabbits (Table S1) were reconstructed by micro-CT of vascular corrosion casts according to the protocol described by Vincent et al. ${ }^{17}$ Idealised tapered and non-tapered thoracic aortic geometries, both neglecting branches, were also constructed for one of the geometries.

\subsection{Geometric analysis}

For all geometries the aortic centreline was determined and curvature and torsion, which respectively describe 3D bending of the aorta and twisting of its plane of curvature, were computed $\left(\mathrm{VMTK}^{18}\right)$. Taper between two locations, which is a measure for the rate of change of radius, was calculated as the arctangent of the difference in radii divided by the distance between the slices, measured along the aortic centreline.

\subsection{Modelling parameters and assumptions}

Simulations of incompressible Newtonian flow were performed using a spectral $/ h p$ element solver. ${ }^{19}$ Steady flow was simulated in all geometries assuming rigid walls. On the basis of experimental mean flow rate measurements ${ }^{20}$ and the observation that mean aortic blood velocity is independent of body mass, ${ }^{21}$ an average aortic root inflow velocity $u_{0}$ of $0.199 \mathrm{~m} / \mathrm{s}$ was assumed for both age groups in the first set of simulations. The inflow Reynolds number Re (indicating the ratio of inertial to viscous forces) for each of these simulations is listed in Table S1, where Re is defined as $\rho \mathrm{u}_{0} \mathrm{D}_{0} / \mu$ with $\rho$ the blood density $\left(1044 \mathrm{~kg} / \mathrm{m}^{3}\right)^{22}, \mu$ the blood dynamic viscosity $(4.043 \mathrm{~g} / \mathrm{ms})^{23}$ and $D_{0}$ the hydraulic diameter ( $=4 \mathrm{x}$ area/perimeter) at the aortic root. To study the influence of geometry alone on the flow, a second simulation was run in each of the anatomically realistic geometries using an inflow Reynolds number of 300. In both sets of simulations, the axial inflow velocity profile was assumed to be plug-like, although parabolic and skewed profiles were also considered for one of the geometries. To analyse the effect of secondary (non-axial) inflow, a $20 \%$ in-plane inflow component pointing away from the centre of curvature was included. (Its direction and magnitude were based on extreme conditions in pigs; unpublished data A. De Luca, 2011.) Flow splits to branches were based on experimental 
measurements, with $14.7 \%$ of the flow in the ascending aorta going to the first branches of the aortic $\operatorname{arch}^{25}$ (the brachiocephalic trunk, left common carotid artery and their branches) and $7.1 \%$ to the left subclavian artery and its branches, ${ }^{25}$ and an estimated total of $2 \%$ of the descending aortic flow going to the 9 or 10 intercostal arteries. ${ }^{17}$ Further flow divisions to the individual branches were calculated using Murray's law. ${ }^{24}$ Parabolic velocity profiles were assumed at the outlets of the branches. At the outlet of the descending aorta, below the fifth pair of intercostal branches, a zero velocity gradient boundary condition was applied. A no-slip condition was prescribed at the arterial wall.

Time-dependent flow dynamics were simulated in 2 immature and 2 mature aortic geometries. In these cases, steady-state boundary conditions at the aortic root and branches were modulated with a velocity waveform that was computed at the proximal descending aorta in a 1D pulse wave propagation model. ${ }^{26}$

\subsection{Haemodynamic mapping}

En face views of the computed WSS distributions in the aortas were generated to facilitate statistical analysis of the results for the different age groups and to allow comparison with lesion distributions. The virtual cutting line was positioned on the ventral side and ensured that the locations of the intercostal ostia were similar to those in previous en face preparations. Around each intercostal branch opening a region of $2.4 \times 3.6 \mathrm{~mm}$ was selected and the WSS data in this region were mapped onto a $20 \times 30$ grid. To obtain the average distribution near the intercostal branches, maps were combined using the centre of the ostium as a datum. Squares close to the ostium were blanked if they lacked data from more than 25 branches (as a consequence of the presence of branch ostia). To obtain the average distribution for the whole aortic segment, each individual 2D map was stretched (using VMTK) to a rectangular shape (with the vertical dimension corresponding to the length along the centreline from the aortic root to just below the 5th intercostal branching pair, and the horizontal dimension corresponding to the perimeter at the aortic inlet) and mapped onto a coarser $20 \times 140$ grid. Values in corresponding grid squares were then averaged for all animals within each age group. Averages were not calculated when data were missing from more than two animals (as a consequence of the presence of branch ostia); these grid squares are coloured white.

\subsection{Statistics}

Data are presented as means with their standard errors (SEM). Geometric outliers were identified using Chauvenet's criterion. Comparisons of geometry and aortic WSS between groups were analysed by Student's unpaired t-test. One-way nested ANOVA was used to assess age-related differences in WSS around intercostal ostia. (Nesting accounted for the fact that each animal contributed 9 or 10 intercostal ostia to the data.) $\mathrm{P}<0.05$ was used as the criterion of significance.

\section{RESULTS}

\subsection{Variation and age-related changes in aortic anatomy}

Figures $1 \mathrm{~A}$ to $1 \mathrm{~J}$ show the luminal surface reconstructions of 5 immature and 5 mature rabbit aortas. The reconstruction approach preserved small-scale features of the geometry, such as sharp branching angles and the cusp near the attachment site of the ligamentum arteriosum. The aortic anatomy varied between rabbits. Most striking was the variation in the branching configuration of the aortic arch: in $50 \%$ of the casts only the brachiocephalic trunk (BCT) and the left subclavian artery (LSA) 


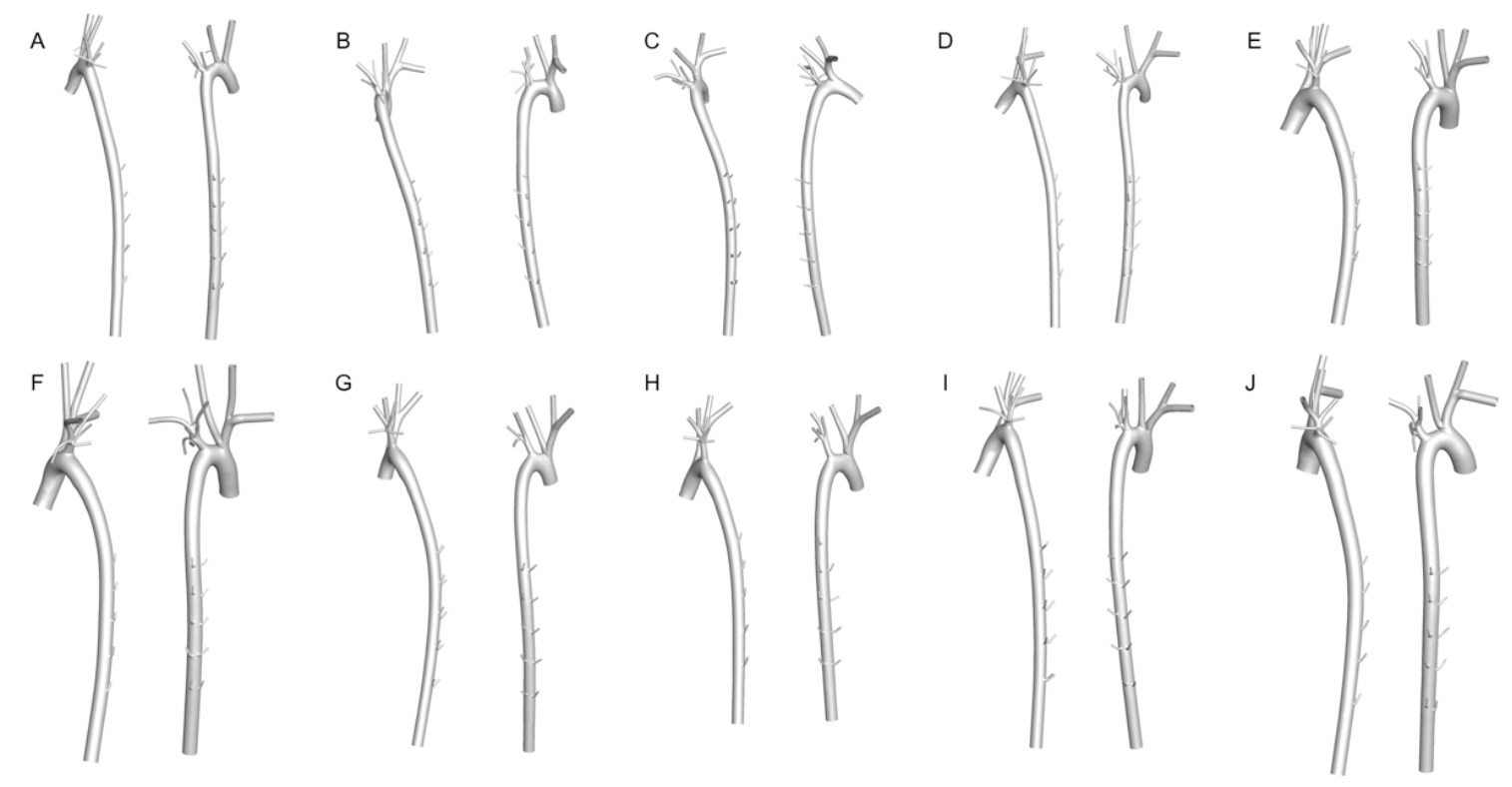

Figure 1: Reconstructions of proximal thoracic aortas of immature $(A-E)$ and mature $(F-J)$ rabbits. All vessels are shown to the same scale following rigid image registration (IRTK, Ixico Ltd). The geometry in $F$ was taken from Vincent et al. ${ }^{17}$

originated from the aortic arch; in one of the geometries the left common carotid artery (LCCA) and the left vertebral artery (LVA) were also aortic arch branches; and the remaining casts had 3 aortic arch branches (LCCA or LVA in addition to BCT and LSA). Moreover, in two cases the most proximal intercostal branch was not paired. Curvature of the aorta increased steadily from the ascending aortic inlet into the aortic arch, reaching its maximum just after the branching point of the brachiocephalic trunk (Figure 2A). The maximum curvature was $(0.946 \pm 0.062) / D_{0}$ (animal-specific values of $D_{0}$ are given in Table S1) and there was no difference in maximum curvature between immature and mature rabbits $(P=0.14)$. At the distal end of the aortic arch, at a distance of $\sim 5 \mathrm{D}_{0}$ along the centreline from the first aortic branch, the aorta was almost straight in some cases, whereas in the descending thoracic aorta curvature was small but generally non-zero. Figure $2 \mathrm{~A}$ also shows the evolution of torsion along the centreline. A common feature was a peak in torsion proximal to or near the branching point of the brachiocephalic trunk. For the descending aorta the concept of torsion is less meaningful because of the small curvature values. As can be appreciated from Figure $2 \mathrm{~A}$, which concentrates on the aortic arch (the range is indicated in Figure 2B), there were no clear age-related differences in the evolution of curvature and torsion.

The inlet diameter $\left(D_{0}\right)$ was $3.96 \pm 0.430 \mathrm{~mm}$ for immature rabbits and $5.23 \pm 0.431$ $\mathrm{mm}$ for mature rabbits $(\mathrm{P}=0.07)$. However, the geometry of one immature aorta (Figure 1D) was more similar to the geometries of the mature group. After excluding this outlier, the inlet diameter for the immature aortas was $3.55 \pm 0.143 \mathrm{~mm}(P=0.01$ on comparison with the mature group). Figure $2 \mathrm{C}$ illustrates the taper of the immature and mature vessels. On average, aortic arches of mature rabbits were more tapered than those of immature rabbits: taper from the aortic root to the first intercostal pair was $0.12 \pm 0.174^{\circ}$ for immature but $0.57 \pm 0.104^{\circ}$ for mature rabbits ( $P=0.058$ for all rabbits; $P=0.003$ without the immature outlier). This difference remained when comparing the fifth intercostal pair with the aortic root $(P=0.13$ for all rabbits; $P=0.018$ without the immature outlier), although taper angles were similar for 


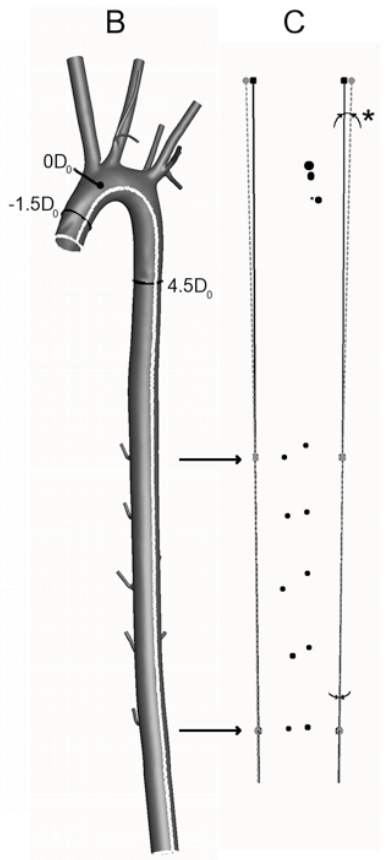

Figure 2: $A$ : Curvature and torsion of the aortic arch of immature and mature rabbits (mean \pm 1 SEM). $B$ : An aortic geometry showing the locations corresponding to the start, zero point and end of the horizontal axis of the plot in $A$, and the cutting line used to open the vessels. $C$ : Diagram of opened immature and mature vessels, scaled longitudinally and circumferentially to match length and perimeter at the first intercostal branch pair respectively, showing approximate branch locations and magnified (x4) taper angles ( ${ }^{*}$ significant without outlier in the immature group)

the descending aortic segment between the first and fifth intercostal pairs $(P \approx 0.5$ with and without the immature outlier).

Since the geometry of Figure 1D was identified as an outlier in the immature group based on Chauvenet's criterion, it was not included in the haemodynamic study.

\subsection{Changes in flow characteristics with age}

In the aortas of all mature rabbits, flow characteristics were similar to those described by Vincent et al. ${ }^{17}$ Briefly, two Dean-type vortices of unequal magnitude formed in the aortic arch and continued to propagate down the thoracic descending aorta; axial velocities in this part of the aorta were skewed to one side. However, in immature rabbits the aortic flow characteristics were different. In particular, although Dean-type vortical structures still formed in the aortic arch, they did not extend into the descending aorta. Representative cases from each age group are shown in Figure $3 A$ and $3 D$, using the $\lambda_{2}$-criterion to define the coherent vortical structures. ${ }^{27}$ To determine the reason for this age-related difference, the CFD models of the representative cases were matched in a stepwise fashion. Matching the Reynolds number of the immature aorta with the mature case (Figure 3B), setting flow splits towards the arch branches to zero (Figure $3 \mathrm{C}$ and $3 \mathrm{E}$ ), and idealising the mature geometry by physically omitting all branches and making its cross-sectional shapes circular (Figure 3F) did not bring the results closer to each other. Only removing taper from the mature model drastically reduced the extent of the vortical structures (Figure 3G), making it similar to the immature case. 


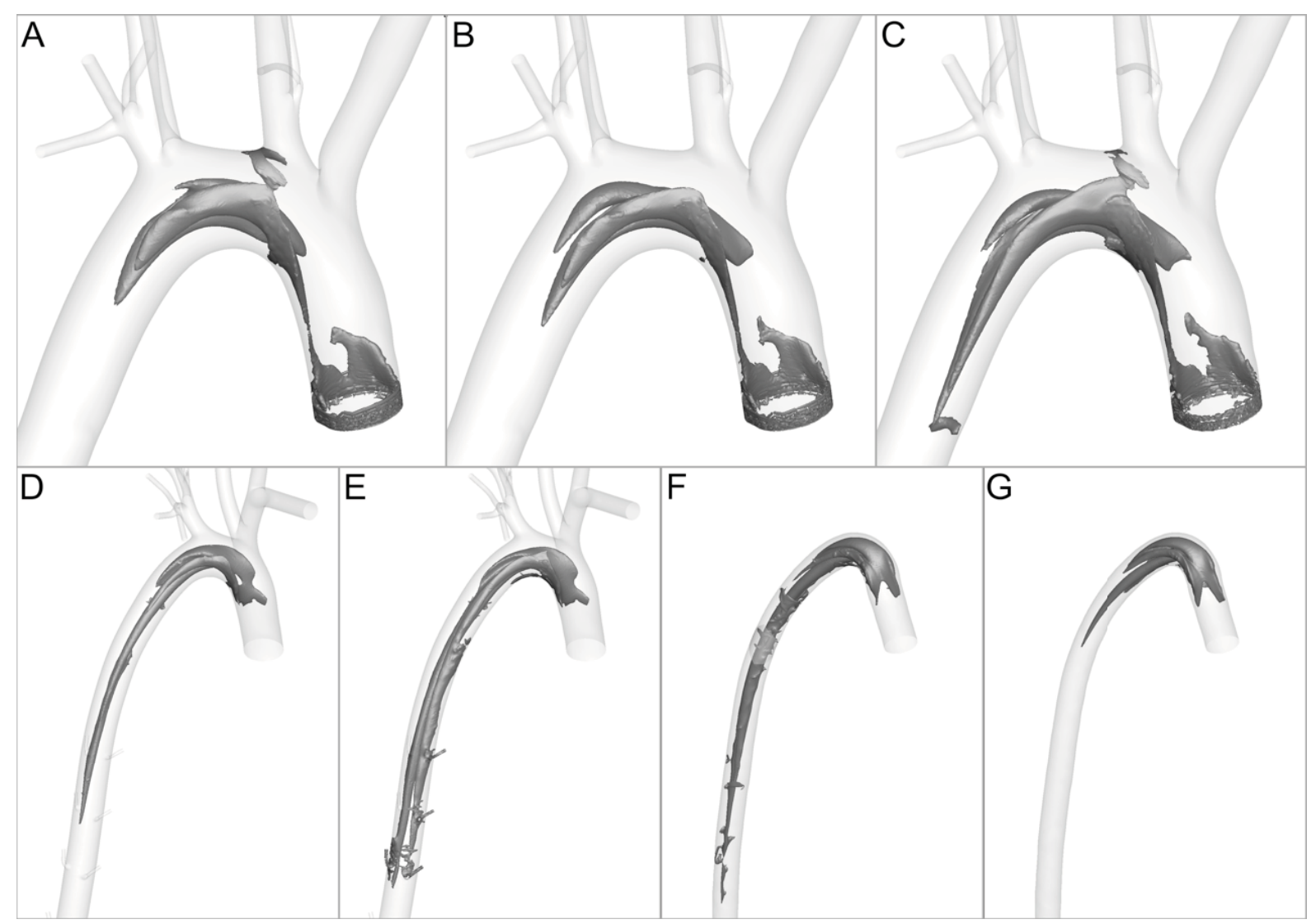

Figure 3: Coherent vortical structures ${ }^{21}$ for the following simulations: $A$ : immature geometry with average inflow velocity $u_{0}=0.199 \mathrm{~m} / \mathrm{s} ; B$ : immature geometry with inflow Reynolds number $\operatorname{Re}=300 ; C$ : immature geometry with $\mathrm{Re}=300$ and zero flow in arch branches; $D$ : mature geometry with $\mathrm{Re}=300$; $E$ : mature geometry with $\mathrm{Re}=300$ and zero flow in arch branches; $F$ : idealised mature geometry with taper $(R e=300)$; $G$ : idealised mature geometry without taper $(R e=300)$. The immature and mature geometries are those of Figures $1 A$ and $1 F$ respectively

\subsection{WSS around intercostal branch ostia}

Figure 4A (top) shows the mean WSS distributions around intercostal ostia in immature and mature rabbits for the simulations with $u_{0}=0.199 \mathrm{~m} / \mathrm{s}$. WSS was highest in a triangular region downstream of the ostium, and was also increased proximally. The evolution of WSS along the longitudinal centreline through the branch (corresponding to the average of the two central columns of squares in the maps) is shown in Figure S1. There was a trend for the percentage difference between the peak WSS on the downstream side compared to the peak WSS on the upstream side along this line to be greater for mature (51.5\%, range: $11.5-99.9 \%)$ animals than for immature (41.4\%, range: $6.94-81.9 \%$ ) animals ( $P=0.10)$. WSS was lower in a ring around the ostium and to its sides. The average WSS in the 2.4x3.6 $\mathrm{mm}$ region around immature ostia was $2.43 \mathrm{~Pa}$ (range: 1.38-3.30 Pa), compared to 2.71 Pa (range: 1.81-4.32 Pa) around mature ostia $(P=0.43)$.

The mean immature and mature non-dimensional WSS distributions around ostia based on the simulations with $R e=300$ are shown in Figure $4 B$ (top). Patterns were similar to those described above, but the difference in magnitude between immature and mature geometries was more pronounced. The average WSS, nondimensionalised using $\rho_{0}{ }^{2}$, was 0.0417 (range: $0.0229-0.0595$ ) around immature ostia and 0.0637 (range: 0.0403-0.1194) around mature ostia $(P=0.055)$. 


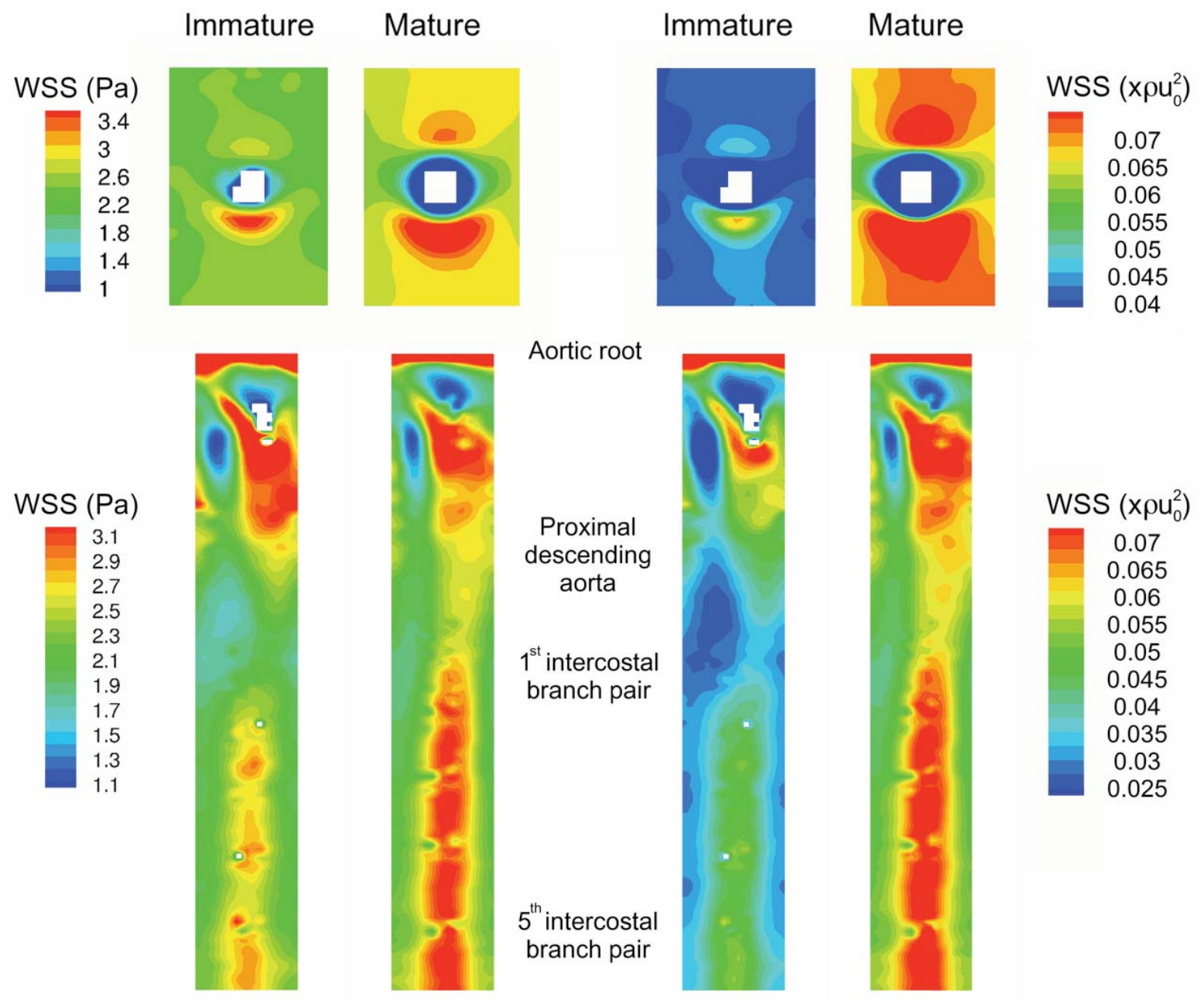

A

B

Figure 4: Average maps of mean wall shear stress in immature and mature geometries around a branch ostium (top) and in the thoracic aorta (bottom), as computed from simulations with average inflow velocity $u_{0}=0.199 \mathrm{~m} / \mathrm{s}(A)$ or inflow Reynolds number $\operatorname{Re}=300(B)$. ( $\rho$ is the blood density.) In $B$, the white spots correspond to locations where ostia were optimally aligned. The luminal surface of each segment, opened ventrally, is shown en face

\subsection{WSS in the thoracic aorta}

Figure 4A (bottom) shows the mean WSS distributions in the thoracic aorta of immature and mature rabbits for the simulations with $u_{0}=0.199 \mathrm{~m} / \mathrm{s}$. WSS was low along the greater curvature of the ascending aorta, on the distal inner wall of the aortic arch and, particularly for immature rabbits, proximal to the superior right intercostal artery. Zones of high WSS were found at the aortic root, where the originally plug-like velocity profile changed rapidly, and distal to the aortic arch branches. In mature animals the latter region extended further down into the descending aorta, resulting in a dorsal streak of high WSS. A similar streak formed more distally in the immature animals. The average WSS was $2.38 \pm 0.23 \mathrm{~Pa}$ in the immature thoracic aortas and $2.47 \pm 0.17 \mathrm{~Pa}$ in the mature aortas $(P>0.5)$.

The mean immature and mature non-dimensional WSS distributions in the thoracic aorta based on the simulations with $\mathrm{Re}=300$ are shown in Figure 4B (bottom).

Patterns were again similar to those for the simulations with constant inflow velocity. 
The average non-dimensional WSS was $0.0405 \pm 0.0041$ in the immature thoracic aortas, and $0.0576 \pm 0.0061$ in the mature aortas $(P=0.06)$.

\subsection{Sensitivity of flow to assumptions and boundary conditions}

A number of additional simulations were run in the geometry of Figure $1 \mathrm{~A}$ to evaluate the sensitivity of the results to modelling assumptions and boundary conditions. In all cases $\mathrm{Re}$ was kept at 300 . Firstly, the effect of the inflow velocity profile was investigated. When a skewed parabolic profile (skewing towards the inner curvature of the aortic arch) was applied at the aortic root, the coherent vortical structure on the anatomical right-hand side developed earlier due to the higher velocity gradient there. For this case, as well as with a parabolic inflow profile, the coherent vortical structures were slightly larger than for the original plug-like profile. However, the WSS distribution was hardly influenced by the changes in inflow profile: patterns were essentially unaltered throughout the aorta, although some minor quantitative changes could be seen in the ascending aorta and aortic arch (Figure S2).

Secondary inflow was generally disregarded. When this assumption was relaxed, WSS increased on the outer wall of the aortic arch, but once more WSS patterns, and the extent of the coherent vortical structures, remained largely unchanged (Figure S3).

The boundary conditions at the aortic arch branches were varied when studying changes in flow characteristics with age, where flow splits to these branches were set to zero (see above). On top of the increase in the extent of the vortical structures (Figure 3B versus 3C), the higher flow split towards the descending aorta caused an increase in the magnitude of the dorsal high WSS streak. However, the location of the streak was not affected.

Finally, unsteady blood flow was simulated in 4 geometries, 2 from each age group, to study the effect of flow pulsatility. The flow waveform and resulting maps of timeaveraged WSS (TAWSS) and the oscillatory shear index (OSI) are shown in Figure S4. With adjusted scaling for each metric, time-averaged patterns were very similar to their steady equivalents. The most notable differences could be found in the proximal part of the descending aorta, where some truly unsteady phenomena occurred (during early diastole there was more secondary flow than with steady-state dynamics in this region). The range of OSI (but not WSS or TAWSS) values in the descending aorta was substantially larger than corresponding values in the aortic arch.

\section{DISCUSSION}

As small changes in geometry can induce significant deviations in WSS, ${ }^{13,17}$ particular attention was paid in this study to the reconstruction of anatomically realistic definitions of the luminal surface. We opted for a post mortem approach combining vascular corrosion casting with micro-CT. This has advantages and disadvantages compared to imaging the lumen in vivo. In vivo techniques are increasingly popular since they allow follow-up. ${ }^{15,28}$ Furthermore, they do not suffer from the effects of resin shrinkage (measured at $<10 \%$ of the diameter ${ }^{29}$ ), which makes cast dimensions smaller than the actual vessels. On the other hand, the cast geometries will not have been affected by (unknown) changes in blood pressure and vessel resistance associated with the use of anaesthetics, ${ }^{30}$ nor by hypervolaemia related to the use of contrast agents. Moreover, the high resolution achieved, with an isometric voxel size of $\sim 50 \mu \mathrm{m}$, would not have been possible using in vivo methods because of interference from surrounding tissue and movement artefacts from 
breathing and the heart beat. The accuracy of the geometries we obtained was exploited maximally by the high-order spectral/hp element flow solver, resulting in very well resolved WSS distribution maps.

The marked variations from the usual branching configuration of the aortic arch, with only 2 branches (BCT and LSA) being present in some animals, are in agreement with a report by Baldwin. ${ }^{31}$ Geometric analysis of the rabbit aortas showed interindividual anatomical variation, but no significant age-related changes, in the curvature and torsion of the aortic arch. However, the aortas of mature animals appeared to taper more than those of immature animals, particularly in the arch region. (Note that there was one outlier in the immature group which was more similar to the mature animals in terms of diameter as well as taper.) The change in taper with age has, to our knowledge, not previously been observed in rabbits. Data on the evolution of diameter along the aortic centreline of younger and older men ${ }^{32}$ suggest that this age-related difference might also hold for the aortic arch of humans.

In each of the geometries, two steady-state simulations were run. Simulations with constant Re allowed us to study direct effects of geometry on blood flow. (When matching the ratio of inertial to viscous forces, as indicated by $\mathrm{Re}$, at the inlets of different arterial geometries, differences in flow characteristics further downstream can be exclusively attributed to differences in the vessel shape). Simulations with constant inflow velocity were more physiologically realistic. Differences in flow dynamics were observed between immature and mature animals: Dean-type coherent vortical structures and the associated offset axial flow, generated by the curvature of the arch, extended further into the descending aorta in the latter. The persistence of the vortical structures can be attributed to the age-related differences in geometric taper of the aortic arch, which accelerates the axial flow and promotes a "stretching" of the vortical structures. Equivalently, this phenomenon can be interpreted using the "ballerina effect": when a rotating body moves closer to the axis of rotation (as when a pirouetting ballerina draws in her arms), it will spin faster in order to conserve angular momentum. In the same way, the greater geometric taper of the mature vessel will cause the vortical structures to rotate faster and hence extend further into the descending aorta. This persistence correlated with a more pronounced dorsal streak of high WSS due to the persisting offset in the axial flow.

Images showing the distribution of lesions in thoracic aortas of individual immature and mature rabbits, obtained by Cremers et al., ${ }^{6}$ are displayed in Figure 5 . Average maps are given by Cremers et al. ${ }^{6}$ We first consider lesions located around the intercostal branch ostia. In immature animals the most diseased region was a triangular zone downstream of the ostium, where we found maximal WSS. There was more variation in the distribution of near-branch lesions in mature animals. Although mean lesion maps ${ }^{6}$ indicated that the lateral margins of ostia are the most athero-prone regions, lesions also developed upstream or downstream in some animals. This contrasts with the mature WSS distribution: the pattern - high WSS downstream and to a lesser extent upstream of the ostium - was similar to the immature one (although the peak in WSS on the downstream side, relative to the upstream peak, tended to be more pronounced, as the taper induced higher local velocities), and was very consistent. 

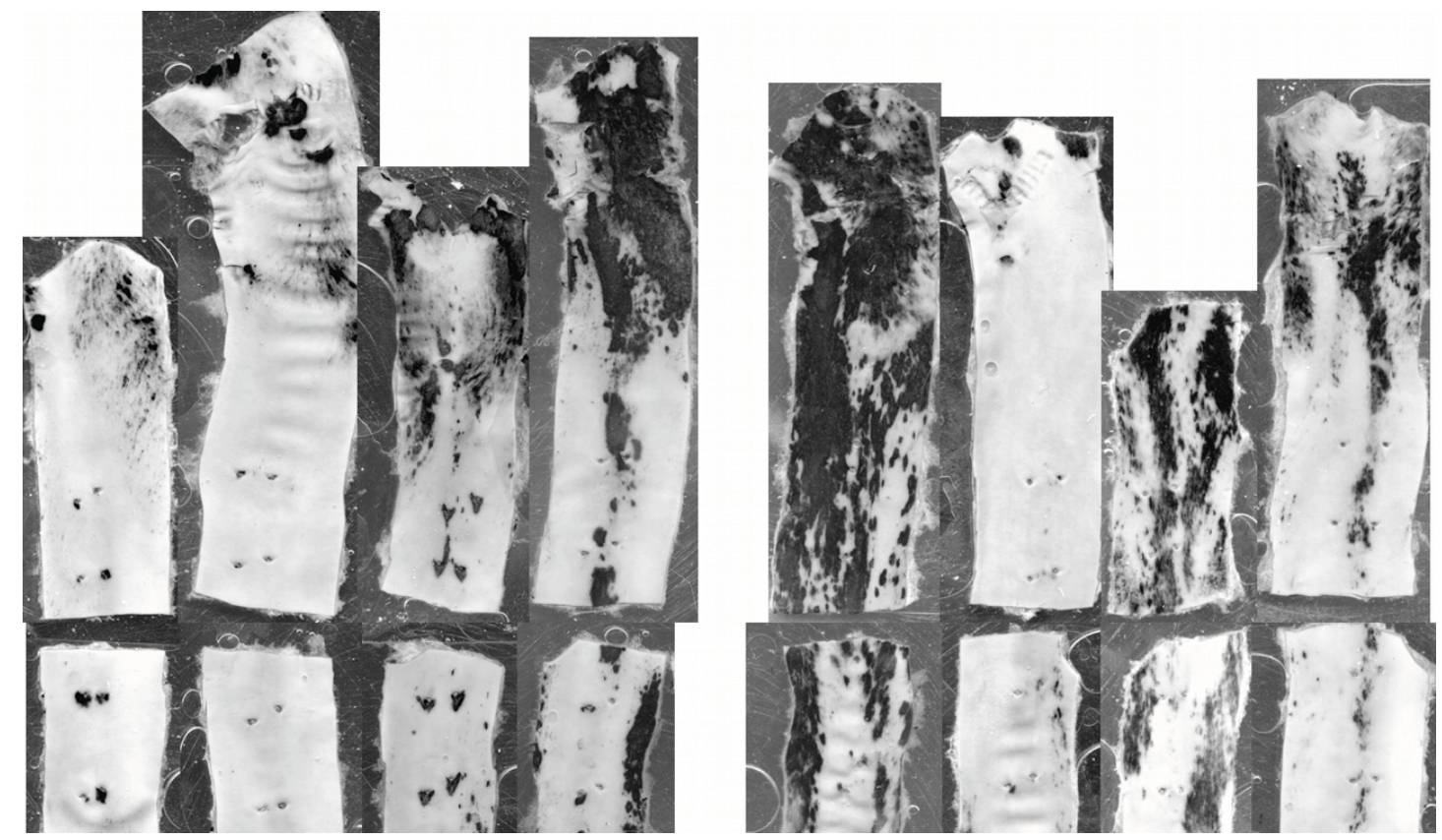

Figure 5: Lesion patterns in the descending thoracic aortas of 4 immature (left) and 4 mature (right) cholesterol-fed rabbits. The luminal surface of each segment, opened ventrally, is shown en face. (The aortas are divided at the third pair of intercostal ostia, which was removed for a different study.) This is a subset of the data from Cremers et al., ${ }^{6}$ showing cases that were most different from each other. Average maps are shown in Cremers et al. ${ }^{6}$

At the level of the first to fifth pairs of intercostal arteries, lesions away from the branches in the descending thoracic aorta are located mainly on the dorsal side (a shift to the ventral side occurring further downstream). This trend is particularly pronounced in mature animals, although outliers exist in both age groups. The dorsal location corresponds to the location of the high WSS streak on our maps. This streak was more pronounced in the mature animals.

There are discrepancies between our CFD results and the data of Bond et al., ${ }^{11}$ who used endothelial nuclear elongation as a surrogate for WSS magnitude. Although both studies indicated elevated WSS downstream of immature branch ostia, only the CFD also indicated elevated WSS upstream, and only the nuclear morphology suggested a reversal in the pattern of WSS with age. (The studies were in better agreement away from branches: both sets of data were consistent with counterrotating helical flows and a dorsal streak of high shear in the descending thoracic segment). One possible explanation is that nuclear morphology reflects factors other than WSS. For example, endothelial cells elongate in response to cyclic stretch as well as shear, ${ }^{33}$ and the elongatory effect of shear can be amplified by flow pulsatility. ${ }^{34}$

A second potential explanation for the discrepancy is that the assumptions underlying the CFD modelling are incorrect. It is important to consider whether their relaxation could change the WSS patterns. A first simplification was the assumption of Newtonian rheology. As quantitative changes introduced by this simplification are only modest, ${ }^{35}$ relaxation of this assumption would not influence our conclusions. Secondly, MRI flow measurements in mice and pigs have revealed that secondary flow is non-zero even at the aortic root and that the velocity profile at this location is skewed rather than plug-like ( ${ }^{36}$; unpublished data A. De Luca, 2011). The sensitivity analysis in the present study showed that more physiologically realistic inflow 
conditions do not substantially alter the WSS patterns. Thirdly, many recent experimental studies highlight the importance of flow reversal as an initiating factor for atherosclerosis, ${ }^{12}$ suggesting that the pulsatility of the flow is decisive for the development of particular disease patterns; an increasing number of computational studies compare lesion distributions against time-dependent flow metrics such as OSI. ${ }^{14,15}$ However, the patterns of TAWSS and OSI we derived from the simulations of unsteady flow in 4 aortic geometries were very similar to the steady WSS patterns. A study of the effects of flow pulsatility and wall compliance on haemodynamics in a mature rabbit aorta (unpublished data A.M. Plata, 2011) also did not reveal any significant change in patterns of time-averaged flow-dependent metrics. On the other hand, it was recently shown for idealised branching geometries that patterns of OSI around ostia can profoundly alter when aortic near-wall flow reverses. ${ }^{16}$ The physiological relevance of these conditions and their effects in anatomically realistic geometries remain to be investigated. Finally, aortic wall motion was not considered in this study. Only a few studies have included this factor in their CFD models; they reported differences in WSS of less than $0.5 \mathrm{~Pa}^{28}$

An early theory of localising factors in atherosclerosis suggested that lesions develop in regions of high WSS. ${ }^{37}$ This view has largely been superseded by the concept that high unidirectional shear stress is protective, lesions instead occurring in areas of low WSS. ${ }^{7}$ Neither of these theories takes into account the age-related changes in lesion location that are now known to occur in human and rabbit aortas. ${ }^{1,3-6}$ The present study does not unequivocally support the low shear theory. Although lesions lateral to branch ostia in mature animals appear to correlate with low shear, lesions downstream of branches in the immature rabbit aorta and lesions away from branches in immature and mature descending aortas seem to occur more frequently in zones of high WSS. Since lesions are also reported to occur downstream of intercostal branches in immature human aortas, ${ }^{1}$ and to be most prevalent on the dorsal wall of the descending thoracic segment of mature human aortas, ${ }^{38}$ the present data cannot a priori be regarded as relevant only to experimental atherosclerosis in laboratory animals; the relation between WSS and lesions at these locations in people requires further investigation.

\section{ACKNOWLEDGEMENTS}

The authors would like to thank Dr. A.M. Plata for useful discussions on computational modelling, and Dr. L.A. Clarke, Dr. Z. Mohri and Mr. A.A.E. Hunt for assistance obtaining the vascular casts. The Barcelona Supercomputing Center is gratefully acknowledged for allowing use of their facilities.

\section{SOURCES OF FUNDING}

This study was funded by the British Heart Foundation Centre of Research Excellence. Flow sensitivity and unsteady simulations were performed under the HPC-EUROPA2 project (project number: 228398) with the support of the European Commission - Capacities Area - Research Infrastructures. Simulations were run on the cx2 HPC system (Imperial College London) and MareNostrum (Barcelona Supercomputing Center).

\section{DISCLOSURES}

None. 


\section{S1 METHODS}

S1.1 Luminal surface reconstruction

All animal procedures complied with the Animals (Scientific Procedures) Act 1986 and were approved by the local ethical review panel of Imperial College London. A total of 7 immature and 6 mature male New Zealand white rabbits (HSDIF strain; Harlan, Bicester, Oxford, UK) were used in this study, but results from only 5 immature and 5 mature rabbits were included in the analysis, for reasons detailed below. These numbers include the mature animal for which flow data were presented by Vincent et al. ${ }^{1}$ Ages and weights of the individual animals are listed in Table S1.

Luminal aortic geometries were reconstructed according to the protocol described by Vincent et al. ${ }^{1}$ Briefly, each rabbit received heparin (2000 USP units, IV) and was subsequently euthanised with an overdose of sodium pentobarbitone (Euthatal, $0.8 \mathrm{ml} / \mathrm{kg}$, IV). The vascular system was flushed with $0.9 \% \mathrm{w} / \mathrm{v}$ saline via a cannula inserted into the left ventricle, with the animal in the supine position. Batson's \#17 resin (Polysciences, Inc.) was injected through the cannula at a pressure of 90 or 100 $\mathrm{mmHg}$, the mean physiological blood pressures for immature and mature rabbits respectively, ${ }^{2}$ until the resin set. After overnight curing of the resin, the carcass was transferred to a $25 \% \mathrm{w} / \mathrm{w} \mathrm{KOH}$ bath to corrode tissues for 2 weeks. The cast was washed with a strong alkaline detergent (Decon 90, Decon Laboratories Ltd) and rinsed with water. Two casts were rejected because the injection pressure could not be maintained, and a third cast because of the presence of a large air bubble on the luminal surface of the descending aorta.

Table S1: Ages (mths=months, wks=weeks) and weights of immature and mature rabbits, together with the voxel sizes for each scan, the total number of elements in the meshed geometries, the hydraulic diameter at the aortic root $\mathrm{D}_{0}$ and the inflow Reynolds number $(\mathrm{Re})$ for simulations with average inflow velocity $u_{0}=0.199 \mathrm{~m} / \mathrm{s}$. Lettering corresponds to Figure 1 . *Geometry $D$ was not included in the haemodynamic analysis

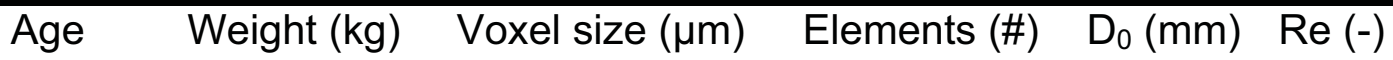

\begin{tabular}{|c|c|c|c|c|c|c|c|}
\hline \multirow{5}{*}{ 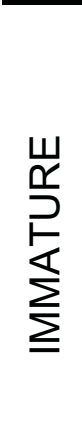 } & $A$ & $11.1 \mathrm{wks}$ & 1.83 & 44.7 & 81164 & 3.35 & 172 \\
\hline & B & 9.9 wks & 1.85 & 48.6 & 81504 & 3.97 & 204 \\
\hline & C & 11.3 wks & 1.83 & 48.6 & 72685 & 3.42 & 176 \\
\hline & D & 9.3 wks & 1.58 & 47.9 & 65988 & 3.44 & * \\
\hline & $E$ & 9.9 wks & 2.02 & 49.8 & 112671 & 5.62 & 289 \\
\hline \multirow{5}{*}{ 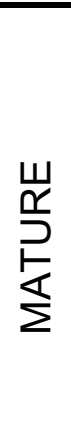 } & $F$ & 18 mths & 3.25 & 55.2 & 79847 & 5.84 & 300 \\
\hline & G & $9.5 \mathrm{mths}$ & 3.24 & 47.7 & 95337 & 4.32 & 222 \\
\hline & $\mathrm{H}$ & $7.6 \mathrm{mths}$ & 3.85 & 50.6 & 98376 & 4.47 & 230 \\
\hline & I & $9.1 \mathrm{mths}$ & 3.76 & 53.3 & 101356 & 4.94 & 254 \\
\hline & $\mathrm{J}$ & $8.7 \mathrm{mths}$ & 4.27 & 49.6 & 91997 & 6.59 & 339 \\
\hline
\end{tabular}


The aortic section of each cast was scanned using a micro-computed tomography (micro-CT) scanner (Metris X-Tek HMX-ST) at a voxel size of $\sim 50 \mu \mathrm{m}$ (see Table 1 for details per cast). This resolution was considered to be sufficient as a close-up scan of an intercostal branch region at half this voxel size did not reveal any smaller geometric details. The resulting DICOM images were segmented and the extracted luminal surface was triangulated and smoothed (Amira, Visage Imaging, Inc.; VMTK, www.vmtk.org; Gambit 2.4.6, ANSYS, Inc.). The surface mesh was then truncated to include only the ascending aorta, the aortic arch with at least its primary branches, and the descending aorta with 5 intercostal branch pairs. (In some cases only a single intercostal artery was present instead of the most proximal intercostal pair). Cylindrical flow extensions were added to the aortic branches and to the descending aortic outlet. A volume mesh for use with a spectral/hp element solver ${ }^{3}$ was generated (TGrid, ANSYS, Inc.) using prismatic boundary elements and tetrahedral internal elements, resulting in a total of $\sim 10^{5}$ spectral elements (see Table S1 for details per mesh). Equivalent meshes in a finiteelement environment would contain $\sim 10^{7}$ linear tetrahedral elements. External faces were curved using SPHERIGON patches. ${ }^{4}$

Idealised tapered and non-tapered thoracic aortic geometries, both neglecting branches, were constructed based on the geometry presented by Vincent et al. ${ }^{1}$ by sweeping a circular section along the centreline of the original geometry (Rhinoceros 4.0 software, Robert McNeel \& Associates). The corresponding volume meshes contained a total of $\sim 1500$ prismatic and tetrahedral elements.

\section{S1.2 Geometric analysis}

For all geometries the centreline of the aorta was traced and resampled using a spline filter $\left(\mathrm{VMTK}^{13}\right)$ so that the final centreline definition was given by a set of points with an inter-point spacing of $D_{0} / 4$. Curvature and torsion of this centreline, which respectively describe 3D bending of the aorta and twisting of its plane of curvature, were computed at the sampling points after application of a Laplacian smoothing filter. For statistical analysis of these parameters between age groups, geometric centrelines were combined by matching consecutive sampling points, using the branching point of the brachiocephalic trunk (as determined with VMTK ${ }^{14}$ ) as a datum.

To analyse tapering of the aortas, the radii of maximum inscribed spheres were computed at the aortic root, near the first intercostal branch pair (or corresponding single branch), and near the fifth intercostal branch pair (VMTK). Note that this metric is only meaningful away from branches. Taper between two locations, which is a measure for the rate of change of radius, was calculated as the arctangent of the difference in radii divided by the distance between the slices, measured along the aortic centreline.

\section{S1.3 Modelling parameters and assumptions}

Flow simulations were performed using a spectral/hp element solver $\left(\mathrm{N} \varepsilon \mathrm{T}_{\mathrm{Tar}} \mathrm{r}^{3,5}\right)$. Assumptions were made to limit the number of model parameters. Firstly, the governing Navier-Stokes equations were reduced by assuming incompressible, Newtonian blood. Secondly, arterial wall compliance and motion were neglected in the present study. The validity of these assumptions is addressed in the Discussion section.

Steady flow was simulated in all geometries. On the basis of experimental mean flow rate measurements ${ }^{6}$ and the observation that mean aortic blood velocity is independent of body mass, ${ }^{7}$ an average aortic root inflow velocity $\mathrm{u}_{0}$ of $0.199 \mathrm{~m} / \mathrm{s}$ was assumed at the aortic root for the first set of simulations. The inflow Reynolds number Re for each of those simulations is listed in Table 1, where Re is defined as $\rho_{0} \mathrm{D}_{0} / \mu$ with $\rho$ the blood density $\left(1044 \mathrm{~kg} / \mathrm{m}^{3}\right)^{8}, \mu$ the blood dynamic viscosity $(4.043$ $\mathrm{g} / \mathrm{ms})^{9}$ and $D_{0}$ the hydraulic diameter $(=4 \mathrm{x}$ area/perimeter) at the aortic root.

When matching the ratio of inertial to viscous forces (as indicated by $\mathrm{Re}$ ) at the inlets of different arterial geometries, differences in flow characteristics further downstream can be exclusively attributed to differences in the vessel shape. Hence, to study the influence of geometry on the flow, a second simulation was run in each of the anatomically realistic geometries using an inflow Reynolds number of 300 , which corresponds to an average inflow velocity of $0.199 \mathrm{~m} / \mathrm{s}$ for the mature geometry presented by Vincent et al.; ${ }^{1}$ WSS for these cases was studied in its non-dimensionalised form (using $\rho_{0}{ }^{2}$ ). In both sets of simulations, the axial inflow velocity profile was assumed to be plug-like, although parabolic and skewed profiles were also considered for one of the geometries. To analyse the effect of secondary (non-axial) inflow, a $20 \%$ in-plane inflow component pointing away from the centre of curvature was included. (Its direction and magnitude were based on extreme conditions in pigs; unpublished data A. De Luca, 2011.) Flow splits to branches for the anatomically realistic geometries were based on experimental measurements, with $14.7 \%$ of the flow in the ascending aorta going to the first branches of the aortic $\operatorname{arch}^{11}$ (the brachiocephalic trunk, left common carotid artery and their branches) and $7.1 \%$ to the left subclavian artery and its branches, ${ }^{11}$ and an estimated total 
of $2 \%$ of the descending aortic flow going to the 9 or 10 intercostal arteries. ${ }^{1}$ Further flow divisions to the individual branches were calculated using Murray's law. ${ }^{10}$ Parabolic velocity profiles were assumed at the outlets of the branches. At the outlet of the descending aorta below the fifth pair of intercostal branches, a zero velocity gradient boundary condition was applied. A no-slip condition was prescribed at the arterial wall. Solutions within each element were approximated with $5^{\text {th }}$ or $7^{\text {th }}$ order polynomials for the anatomically realistic and idealised geometries, respectively. Convergence of the solutions for selected configurations was verified using up to $7^{\text {th }}$ or $9^{\text {th }}$ order polynomials, respectively. The difference in average aortic WSS when increasing the polynomial order from 3 to 5 was below $0.8 \%$ for all steady-state simulations with $\mathrm{Re}=300$.

Time-dependent flow dynamics were simulated in 2 immature and 2 mature aortic geometries (Figure 1 , geometries $A, C, G$ and $J$ ). To damp initial transients two cardiac cycles were simulated. Solutions within each element were approximated using $3^{\text {rd }}$ order polynomials for the first cycle and $5^{\text {th }}$ order polynomials for the second cycle. For the time-dependent simulation, steady-state boundary conditions at the aortic root and branches were modulated using a velocity waveform that was computed at the proximal descending aorta in a 1D pulse wave propagation model. ${ }^{12}$ The waveform was selected at this location to accurately model flow in the descending aorta.

\section{S1.4 Haemodynamic mapping}

En face views of the computed WSS distributions in the aortas were generated to facilitate statistical analysis of the results for the different age groups and to allow comparison with lesion distributions. After removing all branches, each aorta was opened virtually along a cutting line that naturally followed the curve of the vessel $\left(\mathrm{VMTK}^{14}\right)$. The cutting line was positioned on the ventral side and ensured that the locations of the intercostal ostia were similar to those in the en face preparations previously used to map lesions. ${ }^{15}$

Around each intercostal branch opening a region of $2.4 \times 3.6 \mathrm{~mm}$ was selected and the WSS data in this region were mapped onto a $20 \times 30$ grid. To obtain the average distribution near the branches, maps were combined using the centre of the ostium as a datum. Squares close to the ostium were blanked if they lacked data from more than 25 branches (as a consequence of the presence of branch ostia). To obtain the average distribution for the whole aortic segment, each individual 2D map was stretched (using VMTK) to a rectangular shape (with the vertical dimension corresponding to the length along the centreline from the aortic root to just below the 5th intercostal branching pair, and the horizontal dimension corresponding to the perimeter at the aortic inlet) and mapped onto a coarser $20 \times 140$ grid. Values in corresponding grid squares were then averaged for all animals within each age group. Averages were not calculated when data were missing from more than two animals (as a consequence of the presence of branch ostia); these grid squares are coloured white.

\section{S1.5 Statistics}

Data are presented as means with their standard errors (SEM). One immature animal was identified as an outlier by applying Chauvenet's criterion to the hydraulic diameter of the aortic root in each age group. Comparisons of geometry and aortic WSS between groups were analysed by Student's unpaired t-test. One-way nested ANOVA was used to assess age-related differences in WSS around intercostal ostia. (Nesting accounted for the fact that each animal contributed 9 or 10 intercostal ostia to the data.) $\mathrm{P}<0.05$ was used as the criterion of significance.

\section{REFERENCES (for Online Supplement)}

[1] Vincent, PE, Plata, AM, Hunt, AA, et al., Blood flow in the rabbit aortic arch and descending thoracic aorta, J R Soc Interface, 2011;8:1708-1719.

[2] Owen, TL, Effect of age on blood pressure and small vessel reactivity in male rabbits, Blood Vessels, 1986;23:271-278.

[3] Karniadakis, GE and Sherwin, SJ, Spectral/hp Element Methods for Computational Fluid Dynamics, Oxford, UK, Oxford Science Publications, 2005.

[4] Volino, P and Magnenat Thalmann, N, The Spherigon: A simple polygon patch for smoothing quickly your polygonal meshes, In: Werner, B. (ed), Proc. of Computer Animation, Philadelphia, PA, USA, 1998:72-78.

[5] Sherwin, SJ and Karniadakis, GE, Tetrahedral hp finite elements: algorithms and flow simulations, J Comp Phys, 1996;124:14-45.

[6] Avolio, AP, O'Rourke, MF, Mang, K, et al., A comparative study of pulsatile arterial hemodynamics in rabbit and guinea pigs, Am. J. Physiol., 1976;230:868-875.

[7] Weinberg, PD and Ethier, CR, Twenty-fold difference in hemodynamic wall shear stress between murine and human aortas, J Biomech, 2007;40:1594-1598. 
[8] Kenner, T, Leopold, $\mathrm{H}$ and Hinghofer-Szalkay, $\mathrm{H}$, The continuous high-precision measurement of the density of flowing blood, Pflugers Arch, 1977;370:25-29.

[9] Windberger, U, Bartholovitsch, A, Plasenzotti, R, et al., Whole blood viscosity, plasma viscosity and erythrocyte aggregation in nine mammalian species: reference values and comparison of data, Exp. Physiol., 2003;88:431-440.

[10] Murray, CD, The physiological principle of minimum work applied to the angle of branching of arteries, J. Gen. Physiol., 1926;9:835-841.

[11] Barakat, Al, Marini, RP and Colton, CK, Measurement of flow rates through aortic branches in the anesthetized rabbit, Lab. Anim. Sci., 1997;47:184-189.

[12] Alastruey, J, Nagel, SR, Nier, BA, et al., Modelling pulse wave propagation in the rabbit systemic circulation to assess the effects of altered nitric oxide synthesis, J Biomech, 2009;42:21162123.

[13] Antiga, L, Ene-lordache, B and Remuzzi, A, Computational geometry for patient-specific reconstruction and meshing of blood vessels from MR and CT angiography, IEEE Trans Med Imaging, 2003;22:674-684.

[14] Antiga, L and Steinman, DA, Robust and objective decomposition and mapping of bifurcating vessels, IEEE Trans Med Imaging, 2004;23:704-713.

[15] Cremers, SG, Wolffram, SJ and Weinberg, PD, Atheroprotective effects of dietary L-arginine increase with age in cholesterol-fed rabbits, Br. J. Nutrition, 2011;105:1439-1447.

\section{Upstream}

Downstream

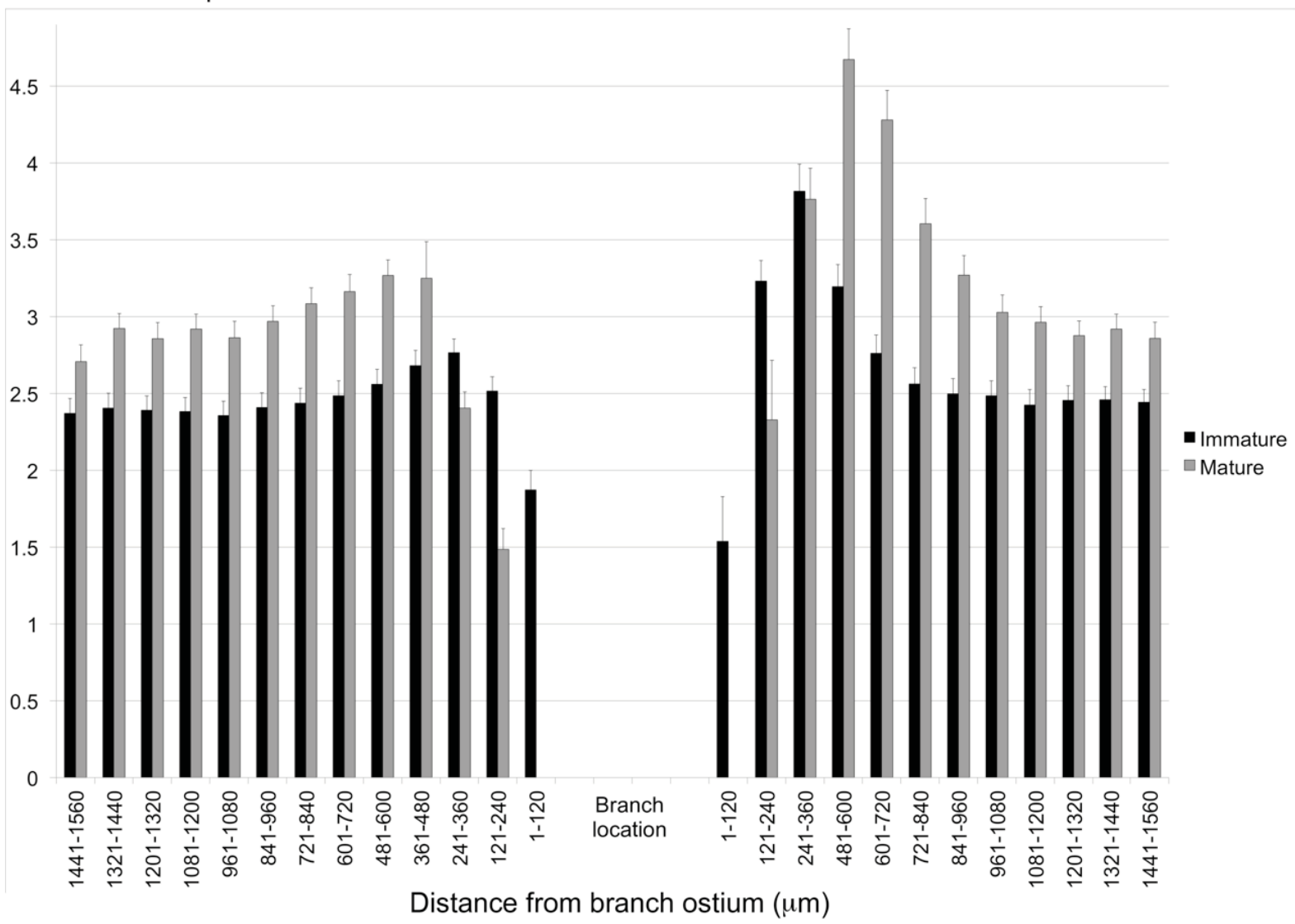

Figure S1: Wall shear stress ( $\mathrm{Pa}$, mean+1 SEM) along the longitudinal line through the center of intercostal branch ostia in immature and mature aortas 


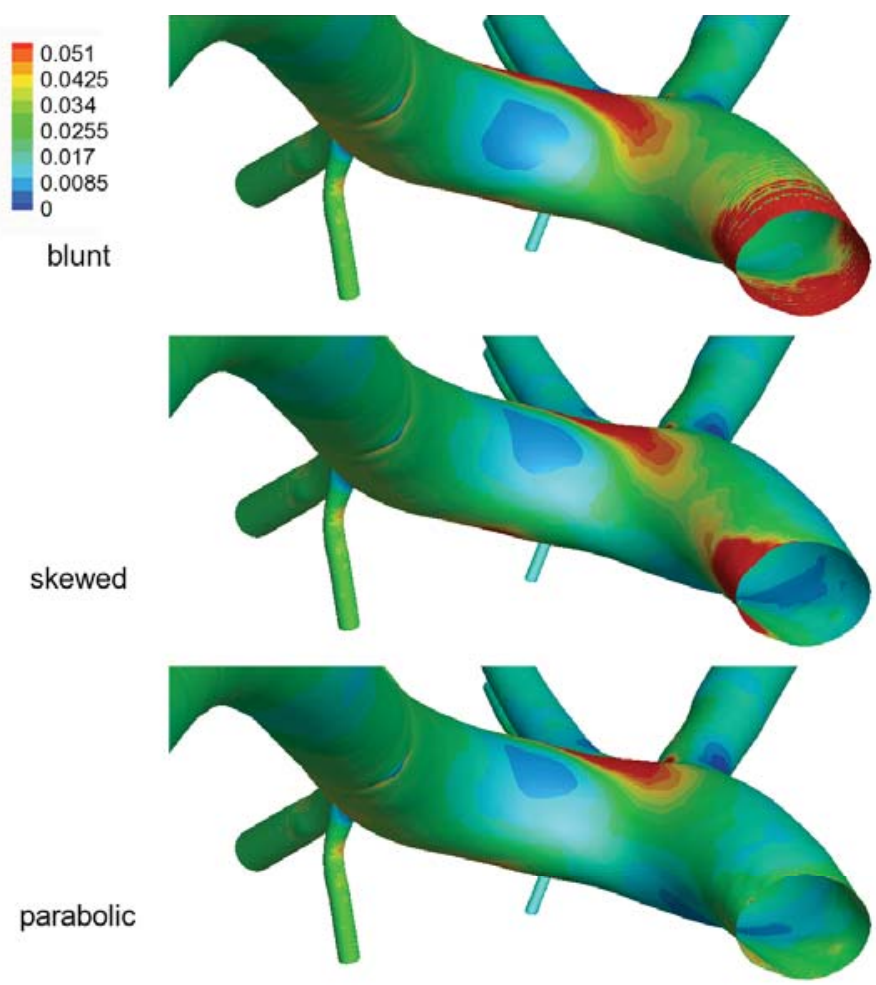

Figure S2: Influence of the velocity profile at the aortic root on wall shear stress in the aortic arch of the geometry shown in Figure 1A (caudal view). The skewed inflow profile was skewed towards the inner curvature of the aortic arch

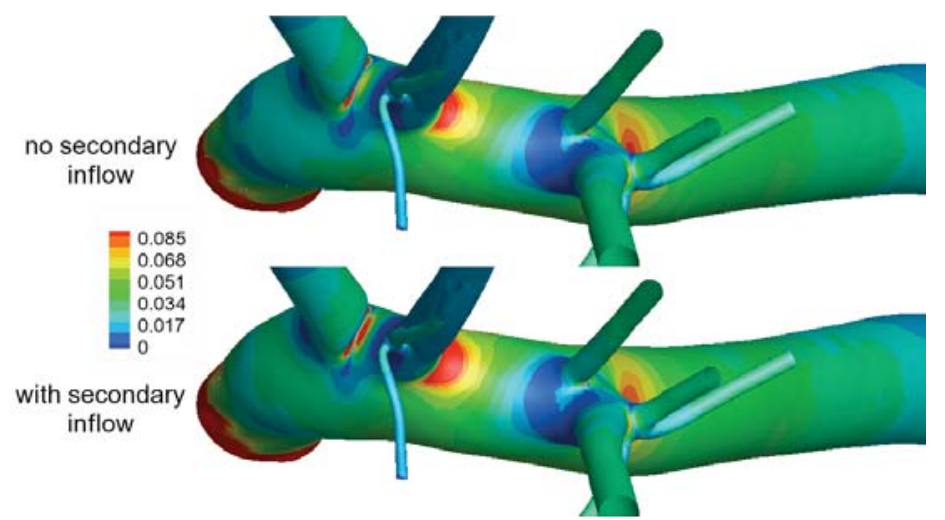

Figure S3: Influence of secondary inflow at the aortic root on wall shear stress in the aortic arch of the geometry shown in Figure $1 A$ (cranial view). An in-plane inflow component of $20 \%$, pointing away from the centre of curvature, was applied for the case with secondary inflow 

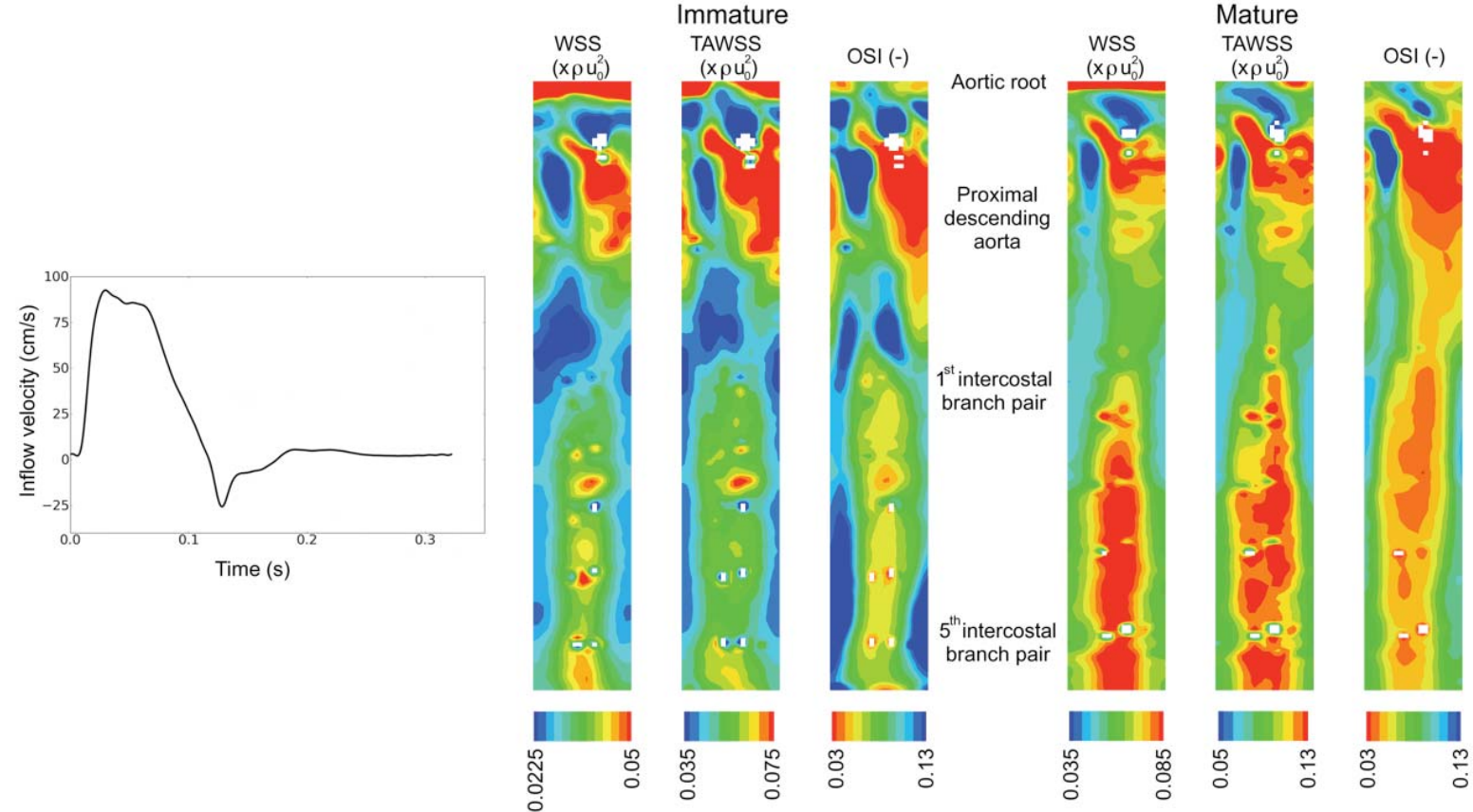

Figure S4: Inflow velocity waveform at the aortic root for time-dependent simulations in 2 immature and 2 mature aortas $(\mathrm{Re}=300)$, and resulting average maps of time-averaged wall shear stress (TAWSS) and oscillatory shear index (OSI). The corresponding average maps of steady wall shear stress (WSS) are also shown. The luminal surface of each segment, opened ventrally, is shown en face 